\title{
A Review on Computational Trust Models for Multi-agent Systems
}

\author{
Gehao $\mathrm{Lu}^{1,2}$, Joan $\mathrm{Lu}^{* 1}$, Shaowen $\mathrm{Yao}^{2}$ and Jim Yip ${ }^{1}$ \\ ${ }^{I}$ School of Computing and Engineering, University of Huddersfield, Huddersfield, UK and ${ }^{2}$ Laboratory of Network In- \\ telligence Computing, University of Yunnan, Kunming, Yunnan, China
}

\begin{abstract}
Trust plays important roles on effective interaction and cooperation for multi-agent systems(MAS). This study aims at finding out the current situation and future trends of computational trustfor multi-agent systems. Through defining seven common compositional elements for the computational trust models, the study points out significant weaknesses in the current design. Finally, the paper figures out the future research trends through discussion and analysis around the strengths and weaknesses identified. Also the paper proposes an idea of using ontology and XML technologies such as $\mathrm{RDF}$ that allow systems to provide both human and machine readable annotations for trust models.
\end{abstract}

\section{INTRODUCTION}

With the increasing size and complexity of the computer systems, it is nearly impossible to design a system from the scratch and control every details of the system purely by human brain (Simon MIT 1996) [1]. It is difficult to control millions of transactions occurred in a large-scale E-market. It is also difficult to monitor an enterprise information system which encompasses huge amount of heterogeneous devices and covers thousands of different geographical locations (Rothkopf AEA 2003) [2]. Grid Computing, Autonomic Computing, Pervasive computing and Multi-agent systems, are all committing themselves to challenge the design of large-scale distributed system (Coulouris AWLP 2000) [3].

Computational trust is to make an intelligent agent trust another agent and delegate part of their tasks to the target agent in a heterogeneous distributed multi-agent environment. Delegation of action is the result of trust and it also forms the foundation of future cooperative large-scale computer systems. Generally, trust toward specific agent is generated through recognition and experience under repeated transactions with that agent. Reputation is the socialized trust which can be propagated through a social network of agents. It helps agents trust the target agent without any direct interaction with the target agent. The benefits of introducing trust and reputation into multi-agent system include:

- As a lubricant, trust can eliminate much of unnecessary communications which are currently necessitates in many interaction protocols thus greatly improve the performance of the multi-agent systems.

- An agent can make decision easier based upon the evaluation of the trustworthiness of another agent. Computational trust is also a very beneficial addition to the traditional decision theory.

\footnotetext{
*Address correspondence to this author at the School of Computing and Engineering, University of Huddersfield, Huddersfield, UK;

E-mail: j.lu@hud.ac.uk
}

- Trust is a kind of soft security which complements the traditional hard security like encryption, authorization, and authentication. An agent exists in complex heterogonous environment must possess both two securities in order to be safe and effective.

The mechanisms for coordinating interactions among agents are always pre-defined, that is, the designer specifies how one agent responses to another agent in a fixed protocol (Ree AGJ 1998) [4]. Such mechanisms are not flexible enough because of the intrinsic highly openness and variability of the distributed systems (Ferber AW 1999) [5]. For example, in open MAS (Multi-agent Systems), an agent cannot expect to always interact with the agents in the predefined application domain in a predetermined way (Subrahmanian MIT 2000) [6]. Agent will interact with different agents coming from heterogeneous applications and they may face challenges from lying, deceiving and accidental incidents (Ferber AW 1999) [5]. Such complexity creates the following questions: can agents accept services from other unfamiliar agents? Can agents make use of the interaction history and transform them into experiences? Can agents avoid excessive negotiation with a familiar agent in an efficient way? Computational trust seems to be the answer and the next step of research for the multi-agent systems. Thus, a systematic review on the existing trust models is necessary.

Trust actually is a belief that someone or agents can delegate the host to finish some actions. There are two layers of meaning in the expression: first, agent should generate the belief of trustworthiness toward some other agents in some specific form; second, agent should make decision whether to delegate actions to the trusted agent. The first layer is actually to study how agents generate and update their belief which is part of research from computational intelligence. The second layer is an extension of the traditional decision theory which adds agents belief, trustworthiness, as one of the concerns during decision making.

There are a few computational trust models and reputation models have been proposed from (Subrahmanian MIT 2000) [6] to (Trung UoS 2006) [7]. From a point of dimen- 
sional view, there are two types of models involved, i.e. local trust based and reputation based models. For local trust based model, the early model developed by Marsh, University of Stirling, 1994, only considers the local trust dimension which only derives trust from the agent's direct interaction without referencing to the recommendations from other witness agents (Marsh UoStirling 1994) [8]. For reputation based models, like SPORA (Zacharia MIT 1999) [9], they only consider the reputation (witness trust) dimension without looking at the local experience of the agent itself. Recently RegreT, Referral Network and TRAVOS take both local trust and witness trust into account (Sabater UAdB 2003) [10] (Luke AAMAS'05 2005) [11]. They combine the value of the two dimensions with relative weights and finally get a sum. Some models, such as FIRE, even introduce additional dimension called as role-based trust and certified reputation (Trung UoS 2006) [7] (Huynh AAMAS 2006) [12].

From a point of algorithmic view, different ways of calculating trust and reputation are proposed. For example, Bayesian systems take binary ratings as input and are based on calculating reputation scores by statistical updating of beta probability density functions (PDF). Such models include TRAVOS (Luke UoS 2006) [13] (Luke AAMAS'05 2005) [11]. Models such as RegreT (Sabater UAdB 2003) [10] (Sabater FWDFTAS 2001) [14] bases on discrete trust model to represent trustworthiness and untrustworthiness as discrete value. A detailed analysis about the composing elements of trust and reputation model will be given in Section 3.

The objective of the study is finding out the current situation and future trends of computational trust for multi-agent systems. It also aims at looking for common necessary compositional elements that compose the models through extracting the essence of those representative models, and summarizing their common weaknesses through comparison, discussion and analysis. Finally, a clear research path is proposed to help the community to promote the research of computational trust.

\section{METHODS EMPLOYED}

The investigation is carried out through viewing and analyzing conference papers, journal papers and technical reports on computational trust from varieties of sources. The criteria of choosing analyzing target are based on whether the model is representative and whether the model reflects the latest trend of the research. To achieve a comparative result, the first thing is to figure out the common basic compositional elements of different models. Taking the elements as parameters then compares the above models in the form of table; it will also try to find out the significance of the result table. The results will be organized in the form of spider graph to show the reader a clear relationship between the evaluation criteria. The study will also differentiate research paths of different models through quantitative analysis and statistics.

\section{RESULTS}

Results are shown from two approaches, i.e. centralized approach and distributed approach. The centralized approach saves all the rating procedure, storage of reputation, query of reputation, searching of comments to the computer server, while the distributed approach finishes all these jobs by agents themselves.

\section{Centralized Approach}

- eBay (eBay WWW 2007) [15]: eBay has built a feedback reputation system for its Customer-to-Customer websites. The goal of designing such a system is to transfer the trust and reputation mechanism in the real life human market to the internet-based e-Market.

- SPORAS (Zacharia MIT 1999) [9]: a reputation model was developed by Zacharia in MIT, 1999. It is an evolved version of the eBay's online reputation models. In this model, only the most recent rating between two users is considered. Another important characteristic is that users with very high reputation values experience much smaller rating changes after each update than users with a low reputation. SPORAS incorporates a measure of the reliability of the users' reputation based on the standard deviation of reputation values.

\section{Distributed Approaches}

- Marsh's Model (Marsh UoStirling 1994) [8]: The pioneer work on computational trust model was done by Mash in 1994. Marsh thought that knowledge, utility, importance, risk, and perceived competence are important aspects related to trust. He defined three types of trust: dispositional trust, general trust and situational trust. The trust management provided by Marsh does not treat the collection of recommendations provided by other agents; he only models direct trust between two agents. The aspect of risk is dealt with explicitly based on costs and benefits of the considered engagement. The decision making is threshold based. Among other parameters the cooperation threshold depends on the perceived risk and competence of the possible interaction partner. If the situational trust is above the value calculated for the cooperation threshold, cooperation will take place otherwise not. Furthermore, the decision making can be extended by the concept of reciprocity, i.e. if one does another one a favor, it is expected to compensate at some time.

- RegreT (Sabater FWDFTAS 2001) [14]: RegreT takes trust as a multi-facet concept and a combination of pieces of information. In RegreT, reputation is a combinatorial product of individual dimension, social dimension, and ontological dimension. The calculation of reputation is same as the calculation in individual dimension. The only difference is that all the reputation under each sub-ontological dimension should be summarized finally. The model deals with three dimensions of trust or reputation. The individual dimension is based on self-made experiences of an agent. The trust values are called direct trust or outcome reputation. The social dimension is based on third party information (witness reputation), the social relationships between agents (neighborhood reputation), and the social role of the agents(system reputation). The ontological dimension helps to transfer trust information between related contexts. For all trust values a measurement of reliability is introduced, which depends on the number of past experience and expected experience (intimate level of interaction), and the variability of the ratings. 
- Referral Reputation (Yu ACM 2002) [18] (Yu ACM 2003) [17]: The underlying computational framework is based on Dempster-Shafer theory (Dempster JRSS 1968) [19]. The model has a well-defined trust network to propagate the trust value from the witnesses, the proposed approach does not concerns the uncertainty surely occurred in the interaction and there is no risk management in this model.

- FIRE (Trung UoS 2006) [7] (Huynh AAMAS 2006) [12]: The FIRE model believes that most of the trust information source can be categorized into the four main sources: direct experience, witness information, role based rules and third party references. FIRE integrates all four sources of information and is able to provide trust metrics in a wide variety of situations. The reliability value bases on the rating reliability and deviation reliability to counteract the uncertainty due to instability of agents.

- TRAVOS (Luke UoS 2006) [13] (Luke AAMAS'05 2005) [11]: TRAVOS (Trust and Reputation model for Agent-based Virtual OrganizationS) is based on probability theory and Bayesian system (DeGroot AW 2002) [20]. The prominent feature of TRAVOS is that it takes confidence into account and the trustor makes decisions based upon the confidence level instead of the trust value. The reputation is not simply added to the direct trust value or confidence value, the introduction of reputation depends on the value of confidence level. If the agent can achieve the minimum confidence level through checking belief of its direct interaction, then it does not have to query other agents. But if the agent cannot find enough confidence, then the agent needs to seek more evidence form the witness.

The researcher builds a test-bed for implementing TROVOS model. Such test-bed is also useful for future evaluation of the researchers own model and the other models. The implementation of the testbed is illustrated in Fig. (1). The test-bed is to mimic a general market where agents carry on business automatically standing for human beings. Each agent seizes some resources and cost specific amount of resources with time elapsing (charging energy). The lifetime of the agent ends when the agent owns nothing (starving to death). The goal of each agent is to maximize their assets (resources) and keep itself alive as long as possible. Transaction is the only way to accumulate resources and avoid dying because the system is designed to make agents achieve more resources in transaction than the resources elapsing with time. There are two attitudes held by the agents: benevolence and malevolence. Under the attitude of benevolence, the agent tends to give full value to the other agent in a transaction; under the attitude of malevolence, the agent tends to give partial value to the other agent in a transaction. The details of transaction are illustrated in Fig. (2).

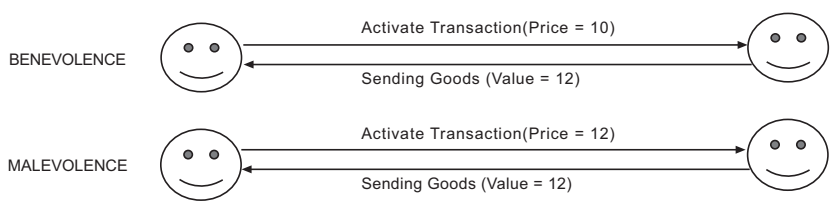

Fig. (1). The benevolence and malevolence agents.

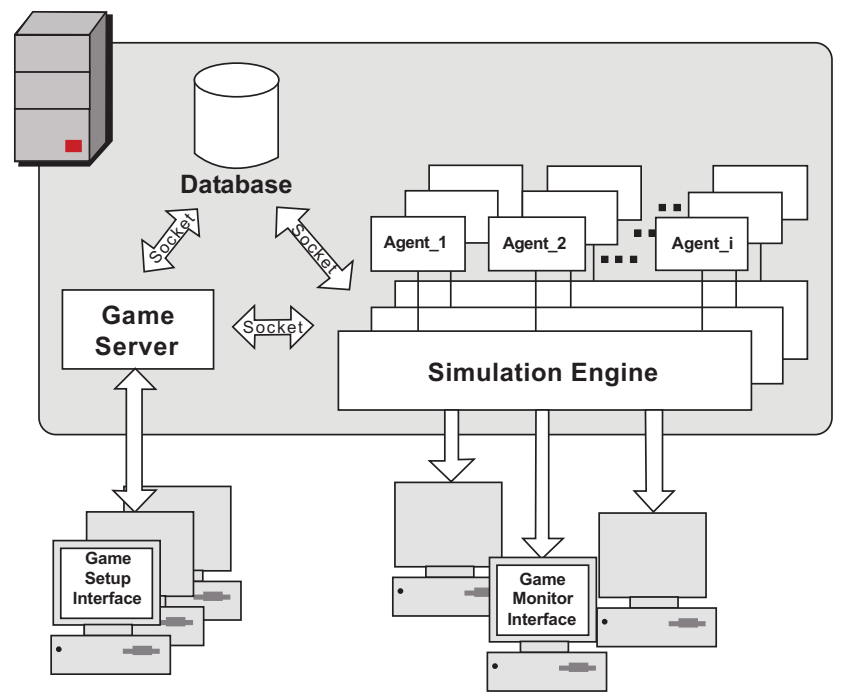

Fig. (2). System design of TROVOS Testbed.

Before any transaction, an agent should find out a target agent and evaluate the trustworthiness of that agent. To find out a target agent, the usual way is to use Contract Net Protocol, the agent broadcast its needs and the other capable agents who are willing to sell goods will response to the request listing their preferred price and quantities. However, in our design, to simplify the scenario and focus on the effect of trust model, the target agent is selected randomly by the simulation engine. The trustworthiness is calculated by the agent through combining the reputation from other agents and the experience accumulated in the past transactions with the target agents. The evaluation engine is based on TRAVOS model. The process of evaluating trustworthiness is illustrated in Fig. (3). Agent must consult other agents through broadcasting request for reputation. If agent has knowledge (experience) about the target agent then the agent should respond to the request and send their experience to originated agent. The originated agent then combine these experience with its own experience according to the TRAVOS model to decide whether to make transaction with the target agent.

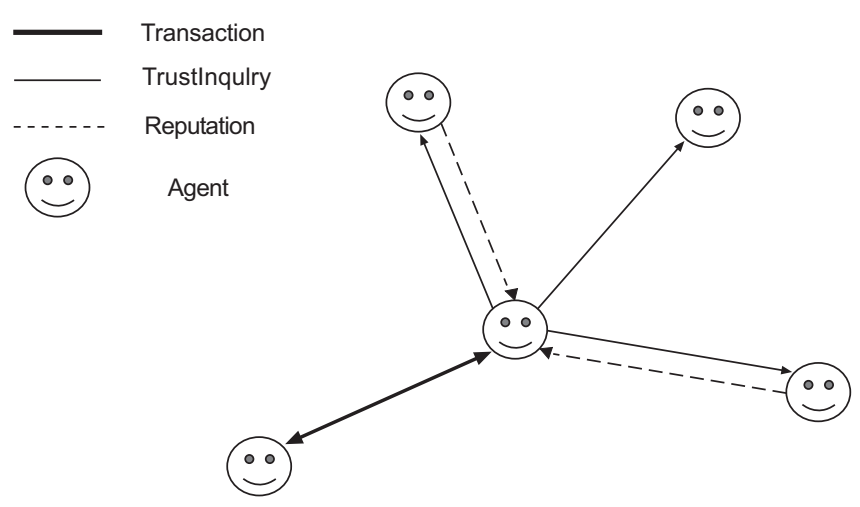

Fig. (3). The agent transaction with trust support.

Through filtering and combining the duplicated terminologies used in the different trust models, seven critical compositional elements can be summarized in Fig. (4). 


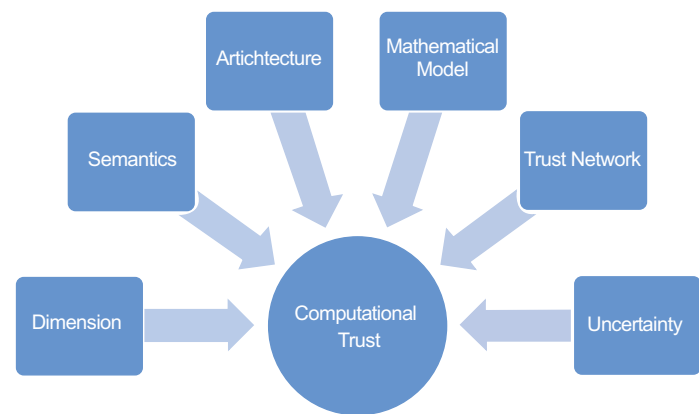

Fig. (4). The compositional elements of computational trust.

- Dimension is to study what the sources of the trust values are.

- The semantics focus on the meaning behind the trust in case that the outcome from the trust is a composite product.

- The mathematical model employed in calculating trust or reputation is sometime called trust computation engine (Josang DSS 2007) [21].

- Trust network is the study on the topology of organizing witness agents and the host agent.

- Uncertainty refers to the management of risk which monitors the accidental incident and environmental changing, and reliability which ensures that the trusted agent is reliable enough even though it is trustworthy based upon the result of mathematical calculation.

The summary of the result is listed in the Fig. (5). Based on the Table in Fig. (5), significant observations can be listed as follow.

1. There are multiple facts (cardinality of dimensions) to forge the trust or reputation. 3 models (eBay,Marsh, SPORAS) are single dimension and 4 models (Referal System, RegreT, FIRE and TRVOS) are multiple dimensions.
2. 6 models presume the semantics behind trust is consistent to all the agents except that RegreT adds an ontological dimension to deal with the semantic difference.

3. 5 models choose distributed architecture rather than centralized architecture (eBay and SPORAS).

4. 6 mathematical models are based on summation or product of different dimensions with selected weights representing their influences. TRAVOS is an exception which is based on Bayesian probability theory.

(The evaluation criterion is the confidence level instead of the trust or reputation value).

5. 5 models, except Referal System and FIRE, don't take trust network and trust transitivity into account.

Their hypothesis is that the trust propagates from the target witness to the host agent without any distortion or loss.

6. None of the models take risk management for accidental incidents and environmental changing into account.

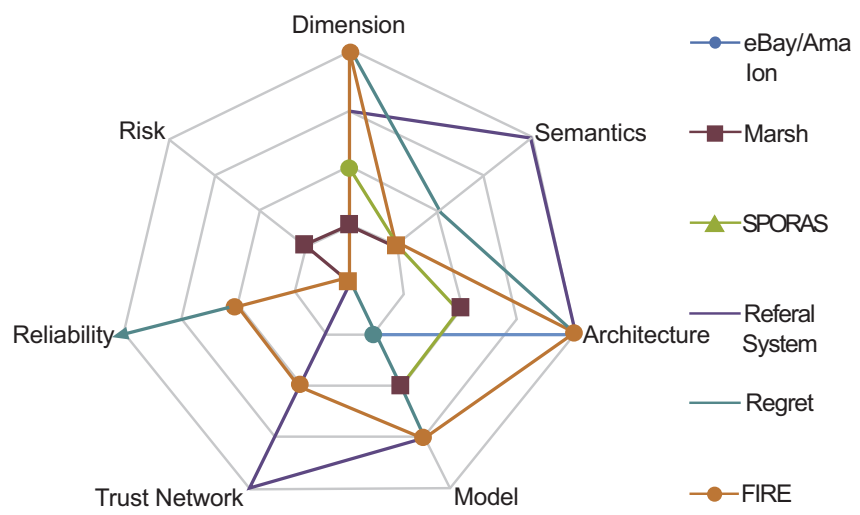

Fig. (6). Strengths and weaknesses of models based upon the elements.

\begin{tabular}{|c|c|c|c|c|c|c|c|}
\hline \multirow[t]{2}{*}{ Model Name } & \multirow[t]{2}{*}{ Dimension } & \multirow[t]{2}{*}{ Semantics } & \multirow[t]{2}{*}{ Architecture } & \multirow[t]{2}{*}{ Mathematical Model } & \multirow{2}{*}{$\begin{array}{l}\text { Trust network } \\
\text { (Trust Transitivity) }\end{array}$} & \multicolumn{2}{|c|}{ Uncertainty } \\
\hline & & & & & & Reliability & Risk \\
\hline eBay [5] & Reputation & Single & Centralized & $\begin{array}{l}\text { Accumulated reputation from feedback of } \\
\text { seller/buyer }\end{array}$ & N/A & N/A & N/A \\
\hline Marsh [7] & Local trust & Single & Distributed & $\mathcal{T}_{z}(y, a)=U_{x}(a) \times \mathcal{I}_{z}(a) \times \widehat{\mathcal{T}_{x}(y)}$ & N/A & N/A & N/A \\
\hline SPORAS [8] & Reputation & Single & Centralized & 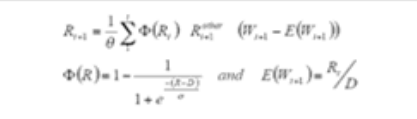 & N/A & Exogenous & N/A \\
\hline $\begin{array}{c}\text { Referal System } \\
{[11,12]}\end{array}$ & $\begin{array}{l}\text { Local trust } \\
\text { reputation }\end{array}$ & Single & Distributed & 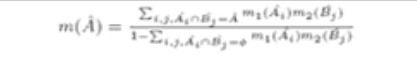 & $\begin{array}{l}\text { Directed graph with } \\
\text { depth limit }\end{array}$ & N/A & N/A \\
\hline RegreT $[9,10]$ & $\begin{array}{l}\text { Local trust } \\
\text { Reputation }\end{array}$ & $\begin{array}{l}\text { Ontology- } \\
\text { based }\end{array}$ & Distributed & 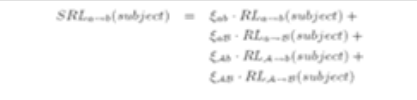 & N/A & Endogenous & N/A \\
\hline $\operatorname{FIRE}[16,17]$ & $\begin{array}{l}\text { Local trust } \\
\text { Reputation } \\
\text { Role-base trust } \\
\text { Certified trust }\end{array}$ & Single & Distributed & $\tau_{K}(a, b, c)=\frac{\sum_{r_{i} \in R_{K}(a, b, c)}{ }^{\omega_{K}\left(r_{i}\right) \cdot e_{j}}}{\sum_{n_{i} \in R_{K}(a, b, c)} \omega_{K}\left(r_{i}\right)}$ & $\begin{array}{l}\text { Direct graph with } \\
\text { depth limit }\end{array}$ & Exogenous & N/A \\
\hline TRAVOS $[14,15]$ & $\begin{array}{l}\text { Local trust } \\
\text { reputation }\end{array}$ & Single & Distributed & 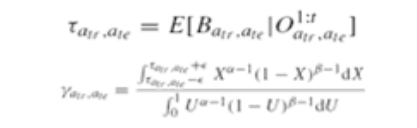 & N/A & Exogenous & N/A \\
\hline
\end{tabular}

Fig. (5). Comparison of different computational trust models. 
The strengths and weaknesses of each model can be visualized through a spider chart in Fig. (6) based upon the compositional elements. The findings are: semantics, risks, trust network pose weak points for most models, whereas the dimension, architecture and mathematical models are intensively studied by researchers.

\section{DISCUSSION AND ANALYSIS}

It is found in Fig. (5) that most models have two basic dimensions: local trust and reputation. But they aren't the only dimensions that can be used to deduce trust. FIRE [7, 12] introduces role-based reputation, which models the trust to the specific role in the society. This is similar to the situation that people always tend to trust some group of people with occupation like professor, doctor or police in the human society. FIRE (Trung UoS 2006) [7] (Huynh AAMAS 2006) [12] also has a design called "certified reputation" which is reputation collected by the trustee from its previous interactions. The truster then does not need to contact its acquaintances to know about the trustee thus the design improves the efficiency of communication. Some idea can be borrowed from social science study on trust; cultural trust and mechanism trust (Sztompka CUP 2000) [22] are trust that may find their places in computational trust.

Most models ignore the semantics behind trust through examining Fig. (5). Their potential hypothesis is that their trust is restricted to a predefined topic. For example, in eBay, trust (reputation) to the seller implicitly means the belief held by the buyer that the seller will send a right product to a right place in a right time (eBay WWW 2007) [15]. However, this is an ambiguous semantic. A few sellers may have believes that a right product to a right place with a little delay deserves the buyer's trust. Such gaps of semantics lead to disruption between buyers and sellers. In multi-agent systems, if two agents with different trust semantics meet, do they simply refuse to trust the other because of incompatible semantics or they need to build up a consensus through negotiation? If the semantics of trust can be adjusted, how often should such adjustment happen and in what extent? It is necessary to create an ontology built upon XML and RDF that allows systems to provide machine-readable semantic annotations for trust of specific domain. The solution is briefly depicted in Fig. (7).

Fig. (6) also shows that most models do not explain how the trust network work and what mechanism the trust transitivity is based. How the trust or reputation value is transferred? Does it simply keep the original value from the witnesses? Or does its value attenuate along distance as same as what happens in the transitivity of human reputation? The solutions to these questions are keys to implement a practical system. Theories about social network analysis in social science make sense to build algorithms for searching witnesses in the network of intelligent agents. The transitivity or propagation algorithm can also benefit from studying the social network analysis. Some researchers have already noticed the transitive trust and proposed their ideas (Josang APCCM 2005) [23].

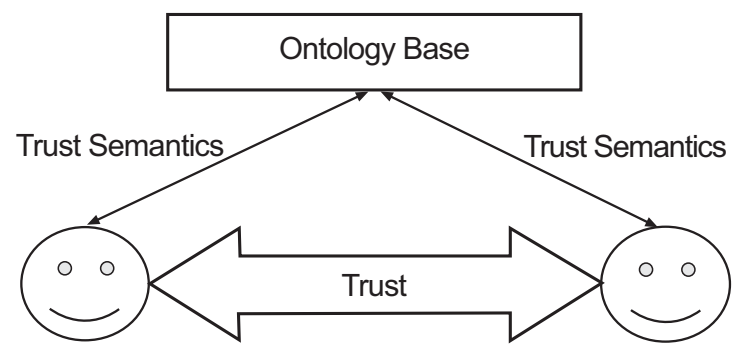

Fig. (7). A possible solution for filling the semantic gap between agents.

Another result from Fig. (5) and Fig. (6) is that none of the reviewed models introduce risk management to control the uncertainty due to the environmental influence or accidental incidents, although they do define reliability to counteract the uncertainty due to instability of individual agent. It is necessary to use risk evaluation to evaluate the risk associated with the prospective transaction under specific environmental facts. The risk here mainly means the risk derivate from the environmental influence or accidental incidents. For example, agent A trust a reliable agent B, but B's environment is instable (B's system often cracks down due to an accidental power off). Such type of risk can be called as environmental reliability. If agent $\mathrm{A}$ has assessed the risk of B's unstable environment additional to its reliability and trust, it will be more careful when making decision about whether to trust or not. Fig. (8) illustrates how the decision of trustworthiness is made under synthesizing the concerns from trust/reputation, reliability and risk.

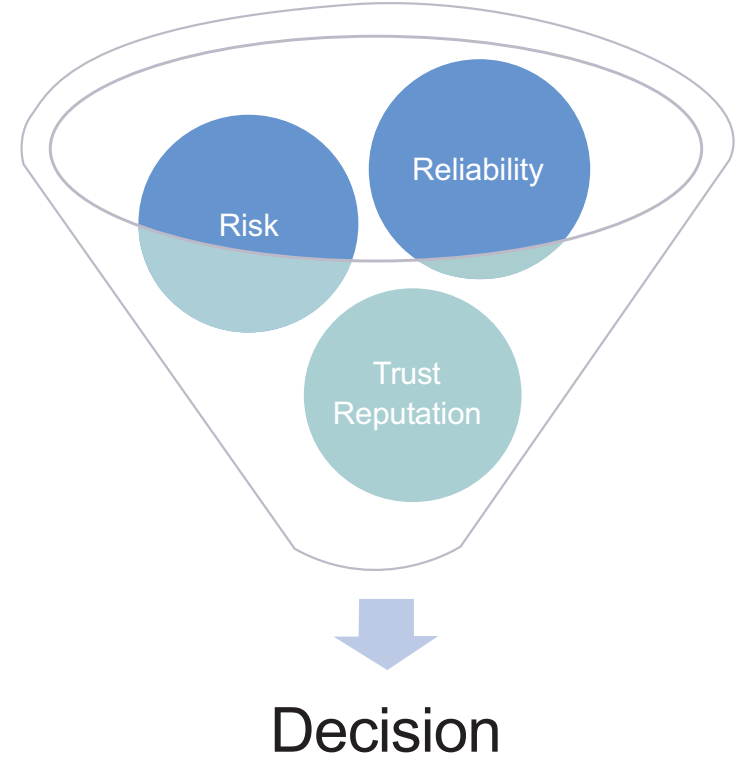

Fig. (8). The decision making process of trust.

An important part is not listed in the comparison table but deserving discussions. Most models stay as a theoretical model without performing a strict experiment in the real system. A few of them do have simple test but not complete. A complete experiment to assess the trust model for MAS should at least pass functionality test, performance test and security test. The functionality test focuses on whether the model supports heterogeneous agents to effectively cooper- 
ate with each other in different scenarios. The performance test focuses on measuring the efficiency of the model in the form of comparison with other models. The security test needs to measure whether the trust model enhances or does harm to the traditional security like authorisation and authentication. The test environment should firstly be a simple but complete multi-agent system. Some tools of building multiagent system can be applied to the experiment like JADE (Java Agent Development Environment), FIPA-OS, zeous, etc. The details about different tools can be seen in technical report from Gerstner Laboratory (Gerstner EASSS'05 2005) [24].

JADE (Bellifemine JWS 2007) [25], FIPA-OS (Guide WWW 2007) [26], and ZEUS (Nwana ACM 1999) [27] are the most prominent and prevalent platforms that support MAS development. The similarities among the three platforms are: all of them are based on Java programming language, all of them are open-source project, and all of them claim to strictly follow the FIPA (Foundation for Intelligent Physical Agents) specification. Through comparing these tools, the following observations and conclusions can be made:

- JADE platform is a better choice for MAS development than FIPA-OS and ZEUS in FIPA compliancy, platform maturity and non-technical concerns.

- Adopting XML as specification message encoding, representation and content language can be a choice to alleviate interaction problems.

- It is necessary to extend the range of applicability of the agent mobility to low-end devices.

- Web Service enhanced agent can freely integrate with any system that supports Web Services; The combination of agent paradigm and the Web Service paradigm may form an intelligent service provision network to complement the traditional static service oriented architectures (SOA).

- It is necessary to introduce agent oriented software engineering process and agent based modeling tools such as AUML (Luke UoS 2006) [13] into MAS platforms.

The continuity of development efforts is important for the maturity of a successful agent platform. For the MAS research community, it is possible to develop better tools and platforms to support the agent development through three routes. The first route is to update the FIPA specification with continuously absorbing new findings and new mechanisms, to incorporate with the existing widely adopted specifications such as XML and UML; the second route is to build more powerful application platform through strengthening the administration, monitoring, debugging and logging functionality, to exploit the successful development tools like eclipse, to incorporate Web Service and agent oriented software engineering; the third route is to maintain and enlarge the open source communities and let more researchers take part in and contribute to the development of platforms.

Fig. (9) is a framework designed for the computational trust and reputation. In the mental space, agents carry out trust learning through observing the results of actions. Such learning leads to the generation, increasing or decreasing of trustworthiness. The learning process is discussed in the next section in details. Next to the mental space is the decision space where agents use trustworthiness derived in the learning process to make delegation decisions. It is combinatory decision making process in that the risk and utility evaluation are also included. The outcomes of specific transaction are constantly observed by the other agents. The observation will become the input of next round trustworthiness learning. Apart from the agents own experience, the trust from the other agents, reputation, is also part of the input of trust learning. The reputation from an organized social network and its propagation mechanism is also research topic in the future work.

Most aforementioned trust and reputation models are mathematically based upon simplistic algebraic summation or explicit statistical deduction through counting the success or failure of historical transactions toward the target agents. Surely these methods can produce reasonable numerical results and then translate them into thresholds which help agents make decisions. However, the assumption that the agents always repeat the same transactions with the same target opponents is impossible in an ever changing complex multi-agent environment.

- First, the semantics are various among objects and it does not make sense to hold some invariable elements as formula to calculate the trustworthy or not trustworthy.

- Second, the count of success or failure of transaction is non-sense when the target of discussion is different. It is impossible to draw an equal mark between a success of a coke transaction and a success of an airplane transaction.

- Third, there is no sure clear border between trustworthiness and distrust-worthiness, that is, trustworthiness or its opposite should be a pattern generated from the repeated transactions instead of a simplistic value.

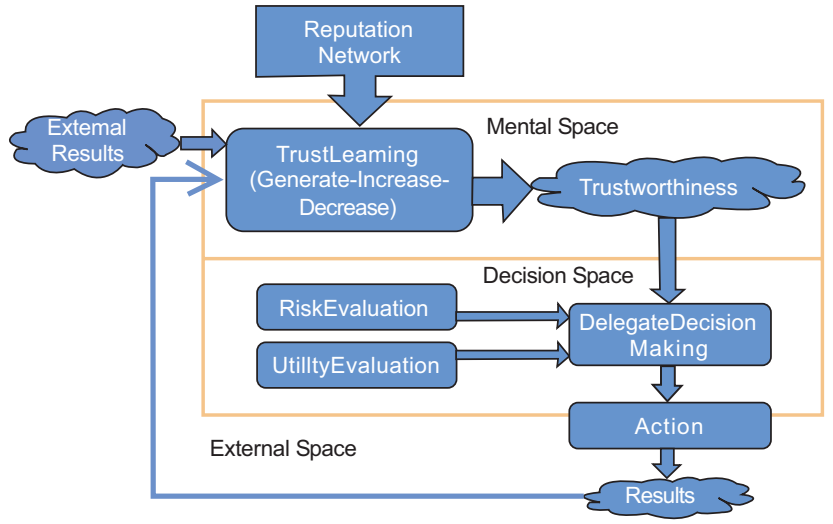

Fig. (9). Strengths and weaknesses of models based upon the elements.

The above analysis leads the modeling of trust and reputation to the area of computational intelligence. Actually, trustworthiness is one kind of belief. The research on generation of trustworthiness is to model a specialized kind of belief for the computer agent. In the study of computational intelligence, several streams are popular and focused in recent years: neural networks, evolutionary computation, swarm intelligence and fuzzy systems. Since 1970s, neural network becomes one of the main research streams of com- 
putational intelligence. It is widely applied in machine learning, pattern recognition, and biological science. The reasons of choosing neural network as the basic calculating mechanism are listed as below:

- Instances are represented by many attribute-value pairs. The target function to be learned is defined over instances that can be described by a vector of predefined features.

- The target function output of the computational trust can be discrete-valued, real-valued or a vector of several real or discrete-valued attributes.

- Neural network learning is good choice for fast evaluation of the learned target function. Usually, the recognition of trustworthiness should be finished several times per second by the agents.

- The ability of humans to understand the learned target function is not important. The weighted learned by neural networks are often difficult for humans to interpret. Fig. (10) illustrates the topological design of the computational trust recognition for intelligent agent. The input layer is composed by dynamically organized element. The elements are extracted from the semantic library according to the target object. For example, if the target object is digital camera, then the elements extracted maybe price, quality, guarantee and delivery. The neural network will gradually adjust its weights in a way of unsupervised learning.

$$
f(x)=K\left(\sum_{i} w_{i} g_{i}(x)\right)
$$

where $\mathrm{K}$ is some predefined function, such as the hyperbolic tangent. It will be convenient for the following to refer to a collection of functions $\mathrm{g}$ as simply a vector $\mathrm{g}=\mathrm{g} 1, \mathrm{~g} 2, \ldots \mathrm{gn}$.

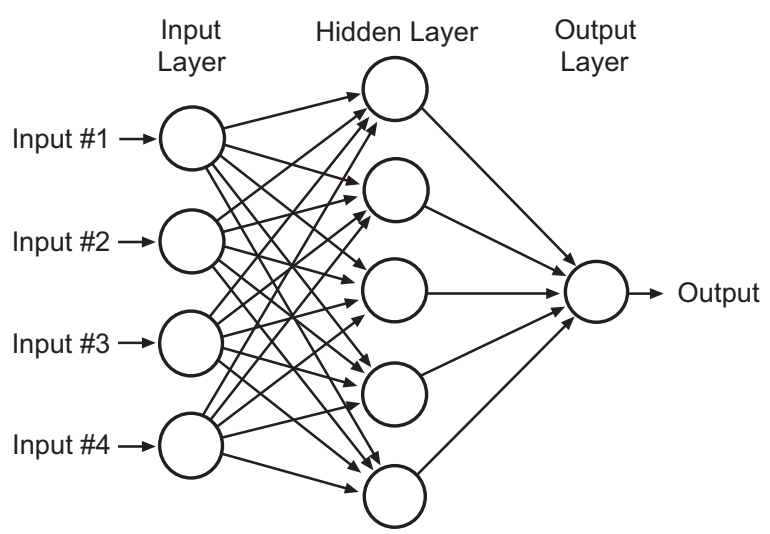

Fig. (10). Neural Network based Trust Calculation.

\section{CONCLUSIONS}

Plenty of interests have been attracted to the construction of computational trust from various research communities. Through analyzing and comprising theses models, the study proposes that a complete computational trust model should at least have seven fundamental elements. Some conclusions are drawn as follows:

- The current trust dimensions are not enough to represent trust in multi-agent systems and new dimension can be modelled through introducing concepts in sociology.
- Agents from different domains must fill their semantic gaps through constructing ontology with XML and RDF.

- The searching algorithm for trust network and the propagation mechanism for trust network can be progressed through introducing techniques in social network analysis.

- Except for the reliability of target agents, agents also need to manage the risk (or environmental reliability) due to environmental changes or accidental incidents.

- A complete experimental platform which used to test the functionality, performance and security of computational trust model is a necessity.

The future work of the research domain can also be naturally derived from the above conclusions.

- The dimension of trust can be extended based on the study of trust in sociology and psychology. It is necessary to categorize the scenarios where the dimension is appropriately used.

- The results in the research of semantic web can be used in the computational trust model. It is necessary to create an ontology built upon XML and RDF that allows systems to provide machinereadable semantic annotations for trust of specific domain.

- Theories about social analysis in social science can be used to build algorithms for searching witnesses in the network of intelligent agents. It is necessary to develop a trust transitivity or propagation mechanism which fulfils the requirement of different situations.

- There is still needs for a simplified, effective mathematical model which can be evaluated through appropriately constructing experiments.

\section{REFERENCES}

[1] Simon HA. The science of artificial 3rd ed. MIT Press 1996.

[2] Rothkopf MH. The future of e-markets. J Econ Lit 2003; 41(1): 214.

[3] Coulouris G, Dollimore J, Kindberg T. Distributed systems: concepts and design. Addison-Wesley Longman Publishing Co., Inc 2000.

[4] Ree Source P. A really great journal paper. Really Great J 1998; 23(2): 728-36.

[5] Ferber J. Multi-agent systems: An Introduction to Distributed Artificial Intelligence.Addison-Wesley 1999.

[6] Subrahmanian VS, Bonatti P, Dix J, et al. Heterogeneous Agent Systems MIT Press 2000.

[7] Huynh TD. Trust and Reputation in Open Multi-agent Systems Ph.D. thesis. Schools of Electronics and Computer Science, University of Southampton 2006.

[8] Marsh S. Formalising trust as a computational concept. Ph.D. thesis. Department of Mathematics and Computer Science, University of Stirling 1994.

[9] Zacharia G. Collaborative reputation mechanisms for online communities. Master's thesis. Massachusetts Institute of Technology 1999.

[10] Sabater J. Trust and reputation for agent societies. PhD thesis. Universitat Autnoma de Barcelona 2003.

[11] Luke TW, Jigar P, Nicholas R, Jennings ML. Coping with inaccurate reputation sources: Experimental analysis of a probabilistic trust model. AAMAS'05 2005.

[12] Huynh TD, Jennings NR, Shadbolt NR. An integrated trust and reputation model for open multiagent systems. Auton Agent Multi Agent Syst 2006; 13: 119-54.

[13] Luke TW. Agent-based trust and reputation in the context of inaccurate information sources. Ph.D. thesis. Schools of Electronics and Computer Science, University of Southampton 2006. 
[14] Sabater J, Sierra C. REGRET: A reputationmodel for gregarious societies. In Fourth workshop on deception fraud and trust in agent societies. Montreal, Canada 2001; pp. 61-70.

[15] eBay Site. (http://www.ebay.com), World Wide Web.

[16] Pesnick P, Zeckhauser R. Trust among strangers in internet transactions: empirical analysis of eBay's reputation system; In: Baye MR, Ed. The economics of the internet and e-commerce; advances in applied microeconomics; Elsevier Science 11: 2002.

[17] Yu B, Singh MP. Searching social networks. In Proceedings of the second international joint conference on autonomous agents and multiagent systems (AAMAS). ACM Press 2003: pp. 65-72.

[18] Yu B, Singh MP. An evidential model of distributed reputation management. In Proceedings of first international joint conference on autonomous agents and multi-agent systems. ACM Press 2002; Vol. 1: pp. 294-301.

[19] Dempster AP. A generalization of Bayesian inference. J R Stat Soc Ser B 1968; 30: 205-47.
[20] DeGroot MH, Schervish MJ. Probability and statistics, 3rd ed. Addison-Wesley 2002.

[21] Josang A, Ismail R, Boyd C. A survey of trust and reputation systems for online service provision. Dec Supp Syst 2007; 43: 618-44.

[22] Piotr Sztompka.Trust: A Sociological Theory (Cambridge Cultural Social Studies). Cambridge University Press 2000.

[23] Josang A, Pope RS. Semantic Constraints for Trust Transitivity. APCCM , University of Newcastle 2005.

[24] Gerstner Laboratory. Agent System Development Hands-on Exercise. EASSS-05 (7th European Agent Systems Summer School), Utrecht, The Netherlands 2005.

[25] Bellifemine F, Caire G, Greenwood D. Developming multi-agent systems with JADE. John Wiley and Sons 2007.

[26] FIPA-OS Developers Guide, http://citeseer.ist.psu.edu/477218.html

[27] Nwana H, Ndumu D, Lee L, Collis J. Zeus: a toolkit and approach for building distributed multi-agent systems. Proceedings of the Third International Conference on Autonomous Agents (Agents'99). ACM Press 1999; pp. 360-1.

(C) Lu et al.; Licensee Bentham Open.

This is an open access article licensed under the terms of the Creative Commons Attribution Non-Commercial License (http://creativecommons.org/ licenses/by-nc/3.0/) which permits unrestricted, non-commercial use, distribution and reproduction in any medium, provided the work is properly cited. 